\title{
Surgical Research
}

\section{The 5 Year Outcomes of Early Postoperative Intra-Peritoneal Chemotherapy (EPIC) in High-Grade Appendiceal Cancer - Does regimen length and cancer subtype make a difference?}

\section{Jasmine Mui" ${ }^{1 *}$ Apoorva Rao', Shoma Barat', Vahan Kepenekian', Winston Liauw², Nayef Alzahrani and Prof David Morris}

${ }^{1}$ Peritonectomy Unit, St George Hospital, Kogarah, 2217, New South Wales, Australia.

${ }^{2}$ Department of Medical Oncology, St George Hospital, Kogarah, 2217, Sydney, New South Wales, Australia.

${ }^{3}$ Department of Surgery, St George Hospital, Kogarah, 2217, New South Wales, Australia; College of Medicine, Imam Muhammad Ibn

*Correspondence:

Jasmine Mui, St George Hospital, Gray St, Kogarah NSW 2217, Australia, Tel: (+61) 432345448 .

Received: 30 August 2021; Accepted: 28 September 2021 Saud Islamic University, Riyadh, Saudi Arabia.

Citation: Mui J, Rao A, Barat S, et al. The 5 Year Outcomes of Early Postoperative Intra-Peritoneal Chemotherapy (EPIC) in High-Grade Appendiceal Cancer - Does regimen length and cancer subtype make a difference? Surg Res. 2021; 3(1): 1-7.

\section{ABSTRACT}

Background: Early Post-operative Intra-Peritoneal Chemotherapy (EPIC) following Cytoreductive Surgery (CRS) and Hyperthermic Intra-Peritoneal Chemotherapy (HIPEC) in high-grade appendiceal peritoneal cancer remains controversial with unclear survival benefit. The following study evaluates the survival outcomes in highgrade appendiceal cancer patients receiving varying days of EPIC in addition to CRS and HIPEC, and if survival varied between different appendiceal cancer subtypes.

Patients and Methods: A monocentric retrospective analysis of patients with high-grade appendiceal cancers managed from 1994 to 2018 was undertaken. An analysis was performed comparing survival between patients who received HIPEC, HIPEC + EPIC, HIPEC + EPIC $<3$ days and HIPEC + EPIC $\geq 3$ days. All patients received CRS.

Results: 212 patients were included in the study. The 5years overall survival was $60 \%$ and $55 \%$ in the HIPEC + EPIC and HIPEC groups respectively. Patients who received $\geq 3$ days of EPIC had an $8 \%$ reduction in risk of death compared to those who had $<3$ days (HR 0.92, CI 0.89-0.94), with a 5-year survival of $62 \%$ in the $\geq 3$ days group compared to $55 \%$ in the $<3$ day group. Patients with signet cell carcinoma had the greatest 5 year survival advantage when both HIPEC and EPIC were given (HR 01.23, CI 01.21-01.26).

Conclusion: EPIC in combination with HIPEC and CRS offers survival benefit at 5years in high-grade appendiceal peritoneal cancer and is most advantageous in signet cell carcinoma. Completing $a \geq 3$ day course of EPIC increases chance of survival at 5years compared to $a<3$ days course.

Keywords

EPIC, HIPEC, High-grade appendiceal cancer, Signet cell cancer.

\section{Abbreviations}

HIPEC: Hyperthermic Intra-Peritoneal Chemotherapy; EPIC: Early Postoperative Intra-Peritoneal Chemotherapy; CRS: Cytoreductive Surgery.

\section{Introduction}

Early Postoperative Intra-Peritoneal Chemotherapy (EPIC) has been an available treatment modality for peritoneal malignancy since the 1990s [1]. Combined with Cytoreductive Surgery (CRS) and Hyperthermic Intra-Peritoneal Chemotherapy (HIPEC), EPIC has been used in the treatment of cancers of ovarian, uterine and bowel pathology [1]. Peritoneal disease offers a distinct set of 
oncological challenges, such as the poor penetration of systemic chemotherapy to peritoneal tumour deposits $[2,3]$. The rationale of EPIC is to target microscopic disease post-CRS and maximise exposure to cytotoxic agents prior to the development of postoperative adhesions [3-5]. This is achieved with cell-cycle specific cytotoxic agents such as 5-fluorouracil or paclitaxel delivered into the peritoneum 23 hours a day for 5 days $[1,6]$.

Appendiceal cancer is one such malignancy typically treated with CRS, HIPEC and EPIC. However, the increased rate of complications in patients who receive EPIC has sparked debate over the overall benefit of EPIC with HIPEC compared to HIPEC alone $[3,5]$. The literature documents grade III/IV post-operative complication rates of up to $58 \%$ in EPIC compared to $20-25 \%$ in HIPEC alone $[3,5,7]$. Furthermore, a systematic review of the literature on EPIC following CRS and HIPEC showed conflicting data regarding long-term overall survival(8). As a result, EPIC has fallen out of favour, with the National Cancer Centre Singapore being one such institute that has ceased to offer EPIC [5].

The current data however may be flawed as many tumour types have been included in analyses regardless of grade and histopathology. As appendiceal cancer has a distinctively different genomic make-up to other malignancies, and as each subtype of appendiceal cancer also has genomic differences, it is important to look at the efficacy of EPIC in these appendiceal subgroups $[9,11]$. Furthermore, grade has also been demonstrated to be an important prognostic factor affecting rates of survival at 5years, with vast differences between low-grade and high-grade disease [3]. Studies from our centre have demonstrated promising results with HIPEC and EPIC in low-grade appendiceal tumours without an increase in significant post-operative complications [12]. The following study aimed to assess overall survival in patients with high-grade appendiceal tumours who underwent CRS and received both EPIC and HIPEC compared to HIPEC alone, and if survival was altered by the number of days of EPIC given or the type of appendiceal cancer.

\section{Methods}

\section{General Methods}

A retrospective analysis was undertaken of prospectively collected data from patients with high-grade appendiceal peritoneal carcinomatosis treated with CRS and HIPEC with and without EPIC at the Peritonectomy Unit of St George Hospital from January 1994 to December 2018.

Patients with histological evidence of high-grade appendiceal cancer were included in the study. They were divided into four groups: HIPEC only, HIPEC + EPIC, HIPEC + EPIC $\geq 3$ days, HIPEC + EPIC $<3$ days

Tumours were further divided into Goblet Cell, Mucinous, Adenocarcinoma and Signet Cell Carcinoma.

\section{Preoperative management}

All patients received preoperative blood tests, imaging, physical examination and nutritional optimisation.

\section{Cytoreductive Surgery}

Disease extent was assessed intra-operatively using the Peritoneal Cancer Index (PCI)(13). CRS was performed using Sugarbaker's technique and all residual disease post-CRS was recorded using the Complete Cytoreduction (CC) score [14].

\section{Hyperthermic Intra-Peritoneal Chemotherapy (HIPEC)}

HIPEC was performed after CRS via the coliseum technique, enabling instillation of heated chemotherapy into the abdomen. Either Mitomycin C $\left(12.5 \mathrm{mg} / \mathrm{m}^{2}\right)$ at 42 degrees for $90 \mathrm{mins}$, or Oxaliplatin $\left(460 \mathrm{mg} / \mathrm{m}^{2}\right)$ at 42 degrees for 60 minutes was administered.

\section{Early Postoperative Intra-Peritoneal Chemotherapy (EPIC)} EPIC was commenced if the patient passed a leak test day 1 postsurgery. This test involved administration of $1 \mathrm{~L}$ of $0.9 \%$ normal saline via an intra-peritoneal Tenkoff Catheter while the intraabdominal drains were clamped. The absence of leakage and major organ failure, as well as the tolerance of the patient to increased intra-abdominal pressure was required to pass the test. EPIC was then commenced day 2 to 6 post operatively via the intraperitoneal catheter. 5-fluorouracil $650 \mathrm{mg} / \mathrm{m}^{2}$ combined with 50 $\mathrm{mEq}$ sodium bicarbonate per day was the regimen of choice, and this was performed in an Intensive Care Unit or High Dependency Unit setting.

\section{Postoperative Management}

Patients were reviewed every three months until 5 years after their operation. For the purpose of this study, the 12-month, 36-month and 5-year follow-up periods were used as defining time points for overall survival. Follow-up involved regular clinical examination, blood tests, imaging and tumour markers when appropriate.

\section{Statistical Analysis}

Data was analysed using IBM ${ }^{\circledR}$ SPSS ${ }^{\circledR}$ software Version 24. Microsoft EXCEL ${ }^{\circledR}$ was used to create graphs, using the KaplanMeier analysis for overall survival as the primary outcome of the CRS + HIPEC with addition of EPIC for selective cases for all appendiceal cancers. Multivariate analysis was carried out using SPSS.

Mean values with corresponding standard deviation for continuous, continuous interval values and median values with corresponding range of values were determined for categorical variables and presented in the patient characteristics table.

The mean value for continuous and continuous interval variables and median value for categorical values were reported at discussion. Incidence and rate of incidence was reported for binary variables and reported as percent, standardised to the $\log$ of $10^{\wedge} 2$. 
Persons who were not marked as dead before the last follow up date were allocated a status of alive at the time of their follow up date. The last follow up date for cases was included in the survival calculations and was marked as "lost to follow up" at that time point. These cases were censored from the overall population at risk group at that time point. Microsoft EXCEL ${ }^{\circledR}$ was used to determine the final status and lost to follow up cases including the survival proportion and probability of survival over time. This was then graphed using the graphing techniques of the same analytical software.

Cox regression method for proportional hazard ratio was used to measure survival probability over time and the Kaplan-Meier technique used to plot and measure survival curve and survival probability.

\section{Results}

\section{Descriptive Characteristics}

There were 212 patients with high-grade appendiceal cancer who underwent CRS and HIPEC from 1994 to 2018. Among them, 146 patients received HIPEC alone while 66 had a combination of HIPEC and EPIC. The most common histological subtype was adenocarcinoma followed by mucinous, signet cell and goblet cell carcinoma. Oxaliplatin was the chemotherapy of choice for HIPEC in the majority of cases (56.13\%) followed by Mitomycin (42.92\%). Complete CRS (0-1) was achieved in $96.23 \%$ of patients. Mean PCI was 22.84, with a median of 25.

\section{Survival Outcomes}

Outcomes were measured using a Kaplan-Meier Curve looking at survival probability over a 5year period (Figure 1). Four groups were plotted on the graph for comparison, these being HIPEC +
EPIC, HIPEC alone, HIPEC + EPIC $<3$ days and HIPEC + EPIC $\geq$ 3 days. HIPEC + EPIC overall compared to HIPEC alone showed a $5 \%$ survival advantage at 5 years $(0.60$ vs 0.55$)$. At 12 months, there was a higher chance of survival in the group who received $\geq$ 3 days of EPIC compared to those who received $<3$ days and those who received HIPEC alone (0.94 compared to 0.87 and 0.88 ). This survival advantage was again present at 5years $(0.62$ for HIPEC + EPIC $\geq 3$ days compared to 0.55 for both HIPEC + EPIC $<3$ days and HIPEC alone) (Figure 1).

In terms of risk of mortality, HIPEC alone had a $12 \%$ increased risk of death at 5years compared to the combination of HIPEC + EPIC (HR 01.12, CI 01.10-01.14). The degree of risk difference is similar if HIPEC $+\geq 3$ days of EPIC was given compared HIPEC alone (HR 0.11, CI 01.09 - 01.13). Meanwhile, HIPEC alone only had a $1 \%$ increase risk of death compared to if HIPEC $+<3$ days of EPIC is given (HR 0.01, CI 00.99-01.04). This indicates the superiority of a $\geq 3$ day regimen. Direct comparison of HIPEC + EPIC $\geq 3$ and HIPEC + EPIC $<3$ groups furthermore demonstrated an $8 \%$ reduction in risk of mortality if three or more days of EPIC was given (HR 00.92, CI 00.89-00.94). Therefore, the group who received EPIC $\geq 3$ days had the greatest effect on decreasing risk of mortality in the combination HIPEC + EPIC group.

\section{Cancer Subtypes}

The survival benefit of EPIC in addition to HIPEC also varies across different cancer subtypes (Table 2). Signet cell carcinoma demonstrated the greatest change in risk of mortality at 5 years if EPIC was given (HR 01.23 for HIPEC vs HIPEC +EPIC, CI 01.21-01.26), while adenocarcinoma showed the smallest change in risk of mortality (HR $01.06 \mathrm{CI} 01.03-01.08$ ). However, comparing signet cell to non-signet cell appendiceal carcinoma

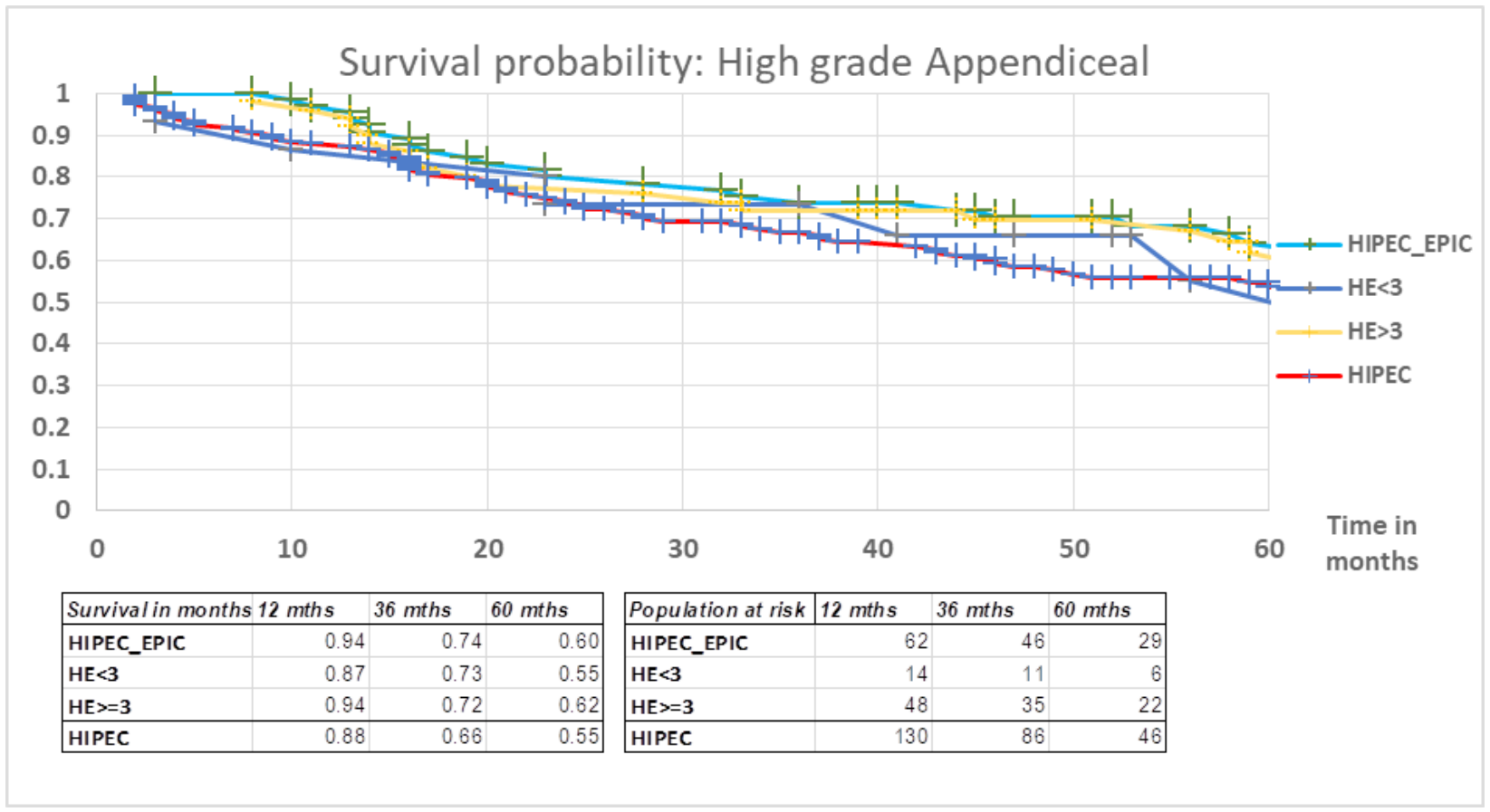

Figure 1: Kaplan-Meier Graph of 5year Survival with HIPEC, HIPEC + EPIC in High-Grade Appendiceal Cancer. 
Table 1: Patient characteristics

\begin{tabular}{|c|c|c|c|c|c|c|}
\hline \multicolumn{2}{|c|}{ Patient Characteristics } & \multirow[b]{2}{*}{$\begin{array}{l}\text { All }(n=212) \\
(p=0.029)\end{array}$} & \multirow[b]{2}{*}{$\begin{array}{l}\text { Goblet Cell }(n=20) \\
(p=0.362)\end{array}$} & \multirow[b]{2}{*}{$\begin{array}{l}\text { Mucinous }(n=136) \\
(p=0.399)\end{array}$} & \multirow[b]{2}{*}{$\begin{array}{l}\text { Adenocarcinoma }(n= \\
\text { 148 })(p=0.076)\end{array}$} & \multirow[b]{2}{*}{$\begin{array}{l}\text { Signet Cell }(n=73) \\
(p=0.869)\end{array}$} \\
\hline Variables & Categories & & & & & \\
\hline \multirow[t]{2}{*}{ Sex } & Male [N (Percent)] & $104(49.06)$ & $9(45.00)$ & $68(50.00)$ & $72(48.65)$ & $31(42.47)$ \\
\hline & Female & $108(50.94)$ & $11(55.00)$ & $68(50.00)$ & $76(51.35)$ & $42(57.53)$ \\
\hline \multirow[t]{2}{*}{ Age } & Years [Mean (SD)] & $54.90(12.14)$ & $53.35(13.08)$ & $55.27(12.07)$ & $54.43(12.26)$ & $53.46(12.78)$ \\
\hline & Median (Range) & $56.03(25.88-81.56)$ & $51.16(36.36-77.18)$ & $57.19(26.41-79.78)$ & $55.14(25.88-77.18)$ & $52.45(27.14-81.56)$ \\
\hline \multirow[t]{6}{*}{ PCI } & Mean (SD) & $22.84(12.23)$ & $16.85(11.64)$ & $26.78(11.04)$ & $23.63(11.19)$ & $24.64(09.61)$ \\
\hline & Median (Range) & $25.00(00.00-39.00)$ & $17.00(00.00-35.00)$ & $30.00(01.00-39.00)$ & $25.00(01.00-39.00)$ & $25.00(04.00-39.00)$ \\
\hline & 0-10 [N (Percent)] & $49(23.11)$ & $7(35.00)$ & $17(12.50)$ & $27(18.24)$ & $6(08.22)$ \\
\hline & $11-20$ & $36(16.98)$ & $5(25.00)$ & $22(16.18)$ & $28(18.92)$ & $16(21.92)$ \\
\hline & $21-30$ & $50(23.58)$ & $5(25.00)$ & $30(22.06)$ & $40(27.03)$ & $28(38.36)$ \\
\hline & $>30$ & $77(36.32)$ & $3(15.00)$ & $67(49.26)$ & $53(35.81)$ & $23(31.51)$ \\
\hline \multirow[t]{3}{*}{ Morbidity Grade* } & Median (Range) & $03.00(00.00-05.00)$ & $02.00(00.00-05.00)$ & $03.00(00.00-05.00)$ & $02.00(00.00-05.00)$ & $02.00(00.00-05.00)$ \\
\hline & $0-2$ [N (Percent)] & $105(49.53)$ & $13(65.00)$ & $59(43.38)$ & $76(51.35)$ & $43(58.90)$ \\
\hline & $3-4$ & $102(48.11)$ & $6(30.00)$ & $74(54.41)$ & $68(45.95)$ & $28(38.36)$ \\
\hline \multirow[t]{3}{*}{ CC Score } & Median (Range) & $00.00(00.00-02.00)$ & $00.00(00.00-01.00)$ & $00.50(00.00-02.00)$ & $00.00(00.00-02.00)$ & $00.00(00.00-02.00)$ \\
\hline & cc0-1 [N (Percent)] & $204(96.23)$ & $20(100.00)$ & $129(94.85)$ & $143(96.62)$ & $71(97.26)$ \\
\hline & $c c 2-3$ & $8(03.77)$ & $0(00.00)$ & $7(05.15)$ & $5(03.38)$ & $2(02.74)$ \\
\hline \multirow[t]{3}{*}{ Chemotherapy** } & Mitomycin C [N (Percent)] & $91(42.92)$ & $6(30.00)$ & $67(49.26)$ & $61(41.22)$ & $23(31.51)$ \\
\hline & Oxaliplatin & $119(56.13)$ & $14(70.00)$ & $68(50.00)$ & $85(57.43)$ & $49(67.12)$ \\
\hline & Cisplatin & $2(00.94)$ & $0(00.00)$ & $1(00.74)$ & $2(01.35)$ & $1(01.37)$ \\
\hline \multirow[t]{2}{*}{ Protocol } & HIPEC only [N (Percent)] & $146(68.87)$ & $17(85.00)$ & $81(59.56)$ & $104(70.27)$ & $57(78.08)$ \\
\hline & HIPEC plus EPIC & $66(31.13)$ & $3(15.00)$ & $55(40.44)$ & $44(29.73)$ & $16(21.92)$ \\
\hline
\end{tabular}

Table 2: Comparison of 5year survival of HIPEC +EPIC for Different Tumour Types in High-Grade Appendiceal Carcinoma.

\begin{tabular}{|l|c|c|c|c|}
\hline $\begin{array}{l}\text { 60-month survival } \\
\text { Hazard ratio }\end{array}$ & All High Grade & Goblet & Mucinous & Adenocarcinoma \\
\hline $\begin{array}{l}\text { HIPEC vs HIPEC+EPIC } \\
\mathbf{9 5 \%} \text { CI) }\end{array}$ & $01.12(01.10-01.14)$ & $*$ & $01.17(01.14-01.19)$ & $01.23(01.21-01.26)$ \\
\hline HIPEC vs HE $>=\mathbf{3}$ & $01.11(01.09-01.13)$ & & $01.55(01.52-01.57)$ & $01.06(01.03-01.08)$ \\
\hline HIPEC vs HE $<\mathbf{3}$ & $01.01(00.99-01.04)$ & & $01.14(01.12-01.16)$ & $00.93(00.90-00.95)$ \\
\hline HE $>=\mathbf{3}$ vs HE $<\mathbf{3}$ & $00.92(00.89-00.94)$ & & $00.74(00.71-00.76)$ & $00.87(00.85-00.90)$ \\
\hline
\end{tabular}

*Not enough numbers for comparative analysis

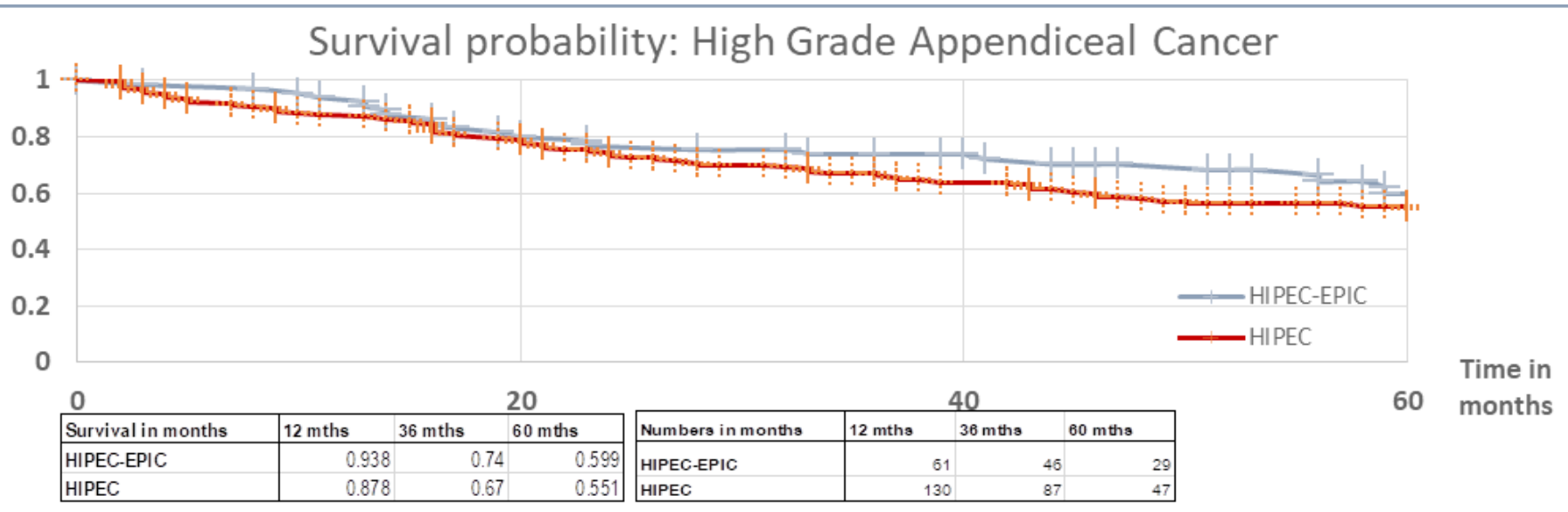

Figure 2: Kaplan-Meier Graph of 5year Survival with HIPEC + EPIC in High-Grade Appendiceal Cancer. 


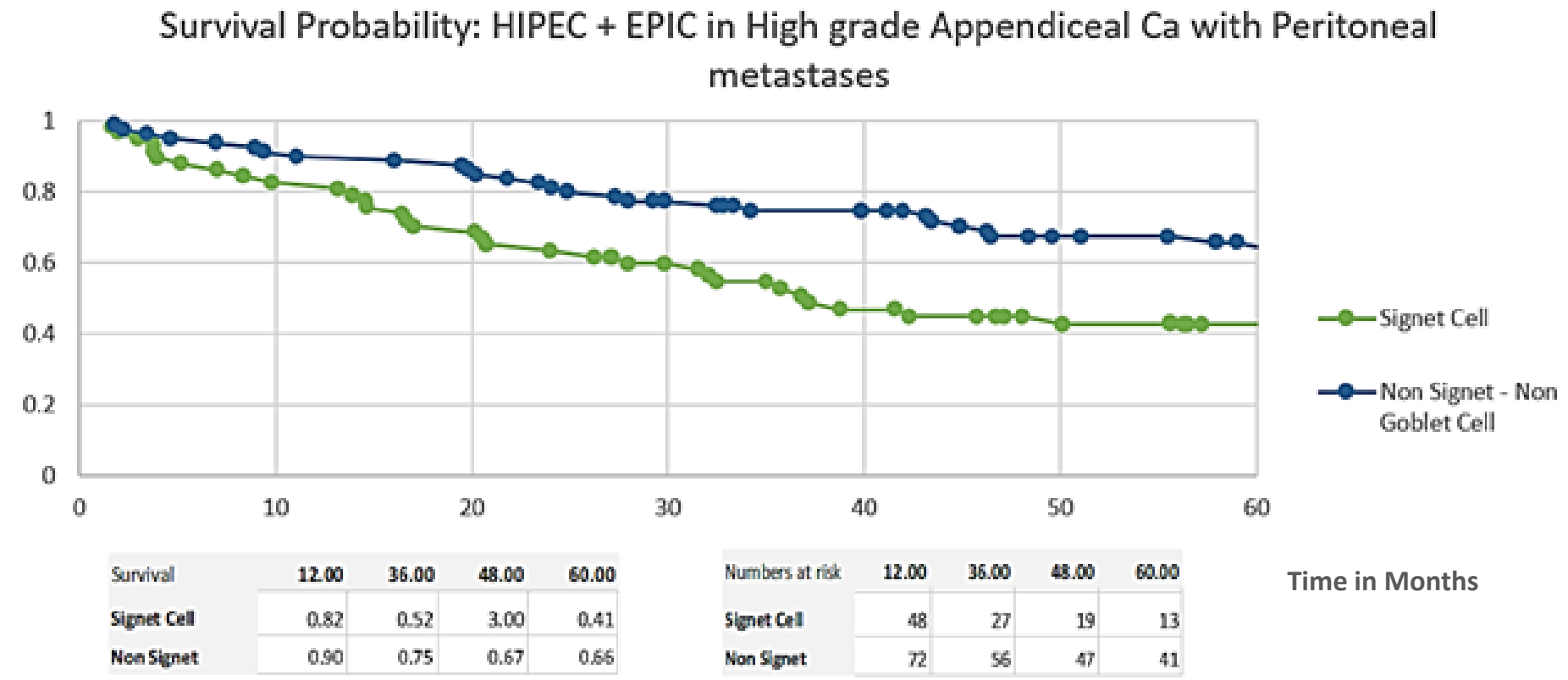

Figure 3: Kaplan Meier Survival of HIPEC + EPIC in Signet compared to Non-Signet Cell Carcinoma.

on the Kaplan-Meier Curve, HIPEC + EPIC demonstrated better overall survival outcomes in non-signet cell cancer, with survival at 5 years being $41 \%$ in the signet cell group compared to $66 \%$ in the non-signet cell group (Figure 3).

Mucinous carcinoma and adenocarcinoma subtypes showed a benefit in HIPEC + EPIC $\geq 3$ days compared to $<3$ days, with a $26 \%$ and $13 \%$ risk reduction in mortality respectively (Table 2 ). There was not enough data to run analyses on patients with goblet cell and signet cell carcinoma for the HIPEC + EPIC $\geq 3$ days and $\mathrm{HIPEC}+\mathrm{EPIC}<3$ days groups.

\section{Discussion}

This study has demonstrated the survival advantage of EPIC in addition to CRS and HIPEC in high-grade appendiceal tumours. In our cohort of high-grade appendiceal cancers who underwent CRS, there was a 5year survival of $60 \%$ in those who had received HIPEC + EPIC compared to $55 \%$ in those who received HIPEC alone. This is comparable to a study performed by Lam et al. who found that in high-grade appendiceal and colorectal cancers, there was a 3 year overall survival of 50\% in the HIPEC + EPIC group compared to $46 \%$ in the HIPEC only group [7].

Comparing data in low-grade appendiceal tumours, Huang et al. reported a 5year survival of $93 \%$ in their patients who had received HIPEC + EPIC, compared to $64.5 \%$ with HIPEC alone, demonstrating a very significant survival benefit in low-grade tumours if EPIC was given. This was less significant in our data in high-grade tumours, with only a $5 \%$ difference in survival at 5years with HIPEC + EPIC compared to HIPEC alone [12]. This difference in survival significance may be due to the slow growing and indolent nature of low-grade tumours which allows for longer survival with disease recurrence compared to high-grade tumours.
The other factor that may contribute to patient mortality is the degree of cytoreduction and PCI. Chua et al. reported that in their study on appendiceal peritoneal carcinomatosis, patients with complete CRS had a greater prognosis for survival at 5years compared to EPIC and HIPEC without CRS [2]. Extrapolating from this, a high PCI preventing complete CRS also leads to poor survival outcomes, with a 5year survival of $12 \%$ if $\mathrm{PCI}>20$ compared to $50 \%$ for PCI $<10$ [7]. Due to the high volume of peritonectomies performed in our centre, complete CRS (C0-1) was achieved in $96 \%$ of patients despite a high mean PCI of 22.84 . Therefore, our data represents the efficacy of EPIC when given in a population with high rates of complete cytoreductive surgery despite high PCI.

The literature has not reported any data on survival outcomes based on number of days of EPIC received, however previous studies on low-grade appendiceal cancer have shown a survival benefit with EPIC even with PCIs greater than 20 [12]. Our centre is currently publishing data on survival outcomes in low-grade appendiceal cancer based on days of EPIC and the results from this preliminary study has shown that in low-grade tumours, less than three days of EPIC offers the same survival advantage as three days or more. The data from this current study suggests that in high-grade tumours, three days or more of EPIC does make a difference to 5year survival, thus grade of tumour is important in determining the most effective length of EPIC.

Histological tumour type also impacts the degree of response to EPIC. In this study, signet cell tumours showed the greatest reduction in risk of mortality out of all the subtypes (HR 01.23, CI 01.21 - 01.26), however the chance of survival is still lower than all the other subtypes combined due to the high mortality associated with signet cell tumours in general. The reason why 
some tumours respond better than others is postulated by Huang et al. In their study of macroscopic tumours in appendiceal adenocarcinoma, patients with hard tumours had a significant decrease in survival. This was explained by the higher presence of signet cells in hard tumours which alters mucin production and structure leading to increased tumour resistance to chemotherapy [6]. This is similar to the findings of our study, where there were worse survival outcomes in signet cell cancers despite response to EPIC. In Huang et al.'s study, soft lesions such as mucinous tumours appeared to be more susceptible to 5-FU and EPIC due to their gelatinous nature allowing penetration of chemotherapy $[4,6,12]$. Mucinous tumours were the second most likely to respond in this study, and this is reflective of previous reports on EPIC efficacy [12]. The better survival outcomes for mucinous tumours may also be a reflection on the nature of the tumour itself in addition to the susceptibility to EPIC, as mucinous tumours do not exhibit lymphatic or haematogenous spread [15].

In our centre, either Mitomycin or Oxaliplatin is used for HIPEC, with the majority of patients receiving Oxaliplatin. Mitomycin is known to have haematological complications such as leukopenia, thus many institutes have altered their HIPEC protocol to transition to other cytotoxic agents such as Oxaliplatin. The implications that this has had on survival has been evaluated in a number of papers and should be discussed in relation to the impact on the outcomes in this present study. Levine et al. found that disease free survival at 3 years in high-grade tumours was similar for both chemotherapy regimens (42.93\% Mitomycin compared to $56.13 \%$ Oxaliplatin) [10]. The haematological toxicity and decreased quality of life however was more significant in the Mitomycin group and toxicity was most severe day 5 to 10 post operation. However, thrombocytopenia was prevalent in the Oxaliplatin group on day 3-8 post HIPEC [10]. Therefore, Oxaliplatin may be more appropriate in patients with leukopenia pre-treatment, while Mitomycin may be safer in patients with thrombocytopenia due to pre-operative chemotherapy [10]. Similarly, other clinical trials suggest non-inferiority of either agent on survival outcomes in the long-term [16-18]. There is limited data demonstrating certain patient groups do better with one agent over the other, such as Oxaliplatin in females with well differentiated colorectal cancer (CRC) and Mitomycin in CRC with low burden of disease and complete cytoreduction, but these results need to be validated with larger multicentre prospective studies [17,19]. Based on the current literature in appendiceal cancer, despite a difference in choice of agent used in our centre, the effect on survival should not be significantly altered between the two groups and therefore this should not have a confounding effect on our results. This has been confirmed by multi-variant analysis.

The largest argument against the use of EPIC is the severity of complications, such as bleeding, pleural effusions, intra-abdominal collections and pneumothorax [2]. This study used the duration in days of EPIC as a measurement of total exposure of chemotherapy in the patient population, therefore those who received more days of EPIC received a higher total dose of 5FU. Higher doses will correlate with greater side effects and while the present study did not look into this specifically, further research to ascertain why EPIC was ceased early and if there were complications, would allow a more robust recommendation to be formulated for the use of EPIC.

\section{Limitations}

There are a number of confounding factors contributing to the significance of the results of this study. The data used was retrospective and may have been incorrectly or inaccurately recorded. Furthermore, the reasons why patients ceased EPIC or had a reduced regimen was not recorded in our current database. This has implications on the clinical applicability of our research. In the literature, up to $50 \%$ of patients prematurely ceased their course of EPIC due to side effects. These included GI bleeding, pneumothoraces and multiple abscesses $[2,3,5,20]$. Poor premorbid function may have also impacted the patient tolerance for EPIC. These factors would be important baseline characteristics to consider when assessing if a patient would be fit to have EPIC. Therefore, we plan to address this in a second paper which reviews reasons for early cessation based on data in our medical records.

As previously mentioned, $96 \%$ of patients in the study had complete cytoreductive surgery. However, the inclusion of those with incomplete cytoreduction may have had an effect on the overall survival analysis and this should be taken into regard when interpreting the results.

Poor patient baseline morbidity and hence being too unfit to qualify for EPIC may explain the poorer survival outcomes in patients who only received HIPEC. The HIPEC alone groups had patients with higher burden of disease, more extensive resections and extensive presurgical treatment, and therefore represent a more morbid population compared to the EPIC group and hence poorer baseline survival [5].

\section{Conclusion}

EPIC in combination with HIPEC and CRS offers a survival benefit at 5years in high-grade appendiceal tumours and this is most advantageous in signet cell carcinoma. Completing a $\geq 3$ day course of EPIC increases chance of survival compared to a $<3$ days course, therefore a full 5 day regimen of EPIC is still recommended in high-grade appendiceal cancer to maximal survival benefits.

\section{Acknowledgements}

The authors would like to acknowledge the members of the Peritonectomy Unit for collection of data over the years, and support in assisting ongoing research in this field.

\section{References}

1. Lemoine L, Sugarbaker P, Van der Speeten K. Drugs, doses, and durations of intraperitoneal chemotherapy: standardising HIPEC and EPIC for colorectal, appendiceal, gastric, ovarian peritoneal surface malignancies and peritoneal mesothelioma. International Journal of Hyperthermia. 2017; 33: 582-592. 
2. Chua TC, Liauw W, Zhao J, et al. Comparative analysis of perioperative intraperitoneal chemotherapy regimen in appendiceal and colorectal peritoneal carcinomatosis. International Journal of Clinical Oncology. 2013; 18: 439-446.

3. Sideris L, Mitchell A, Drolet P, et al. Surgical cytoreduction and intraperitoneal chemotherapy for peritoneal carcinomatosis arising from the appendix. Can J Surg. 2009; 52: 135-1341.

4. Goodman MD, McPartland S, Detelich D, et al. Chemotherapy for intraperitoneal use: a review of hyperthermic intraperitoneal chemotherapy and early post-operative intraperitoneal chemotherapy. Journal of Gastrointestinal Oncology. 2015; 7 : 45-57.

5. Tan GHC, Ong WS, Chia CS, et al. Does early post-operative intraperitoneal chemotherapy (EPIC) for patients treated with cytoreductive surgery and hyperthermic intraperitoneal chemotherapy (HIPEC) make a difference? International Journal of Hyperthermia. 2016; 32: 281-288.

6. Huang Y, Alzahrani NA, Fisher OM, et al. Intraoperative macroscopic tumour consistency is associated with overall survival after cytoreductive surgery and intraperitoneal chemotherapy for appendiceal adenocarcinoma with peritoneal metastases: A retrospective observational study. The American Journal of Surgery. 2019; 217: 704-712.

7. Lam JY, McConnell YJ, Rivard JD, et al. Hyperthermic intraperitoneal chemotherapy + early postoperative intraperitoneal chemotherapy versus hyperthermic intraperitoneal chemotherapy alone: assessment of survival outcomes for colorectal and high-grade appendiceal peritoneal carcinomatosis. The American Journal of Surgery. 2015; 210 : 424-430.

8. Soucisse ML, Liauw W, Hicks G, et al. Early postoperative intraperitoneal chemotherapy for lower gastrointestinal neoplasms with peritoneal metastasis: a systematic review and critical analysis. Pleura Peritoneum. 2019; 4: 20190007.

9. Ang CS-P, Shen JP, Hardy-Abeloos CJ, et al. Genomic Landscape of Appendiceal Neoplasms. JCO Precision Oncology. 2018; 1-18.

10. Levine EA, Votanopoulos KI, Shen P, et al. A Multicenter Randomized Trial to Evaluate Hematologic Toxicities after Hyperthermic Intraperitoneal Chemotherapy with Oxaliplatin or Mitomycin in Patients with Appendiceal Tumors. J Am Coll Surg. 2018; 226: 434-443.
11. Bignell M, Carr NJ, Mohamed F. Pathophysiology and classification of pseudomyxoma peritonei. Pleura Peritoneum. 2016; 1: 3-13.

12. Huang Y, Alzahrani NA, Liauw W, et al. Early Postoperative Intraperitoneal Chemotherapy for Low-Grade Appendiceal Mucinous Neoplasms with Pseudomyxoma Peritonei: Is it Beneficial? Annals of Surgical Oncology. 2017; 24: 176-1783.

13. Tentes AA, Tripsiannis G, Markakidis SK, et al. Peritoneal cancer index: a prognostic indicator of survival in advanced ovarian cancer. European journal of surgical oncology: the journal of the European Society of Surgical Oncology and the British Association of Surgical Oncology. 2003; 29: 69-73.

14. Dubé P, Sideris L, Law C, et al. Guidelines on the use of cytoreductive surgery and hyperthermic intraperitoneal chemotherapy in patients with peritoneal surface malignancy arising from colorectal or appendiceal neoplasms. Curr Oncol. 2015; 22: e100-e112.

15. Ruoff C, Hanna L, Zhi W, et al. Cancers of the appendix: review of the literatures. ISRN Oncol. 2011; 2011: 728579.

16. Hompes D, D'Hoore A, Wolthuis A, et al. The use of Oxaliplatin or Mitomycin C in HIPEC treatment for peritoneal carcinomatosis from colorectal cancer: a comparative study. Journal of surgical oncology. 2014; 109: 527-532.

17. Prada-Villaverde A, Esquivel J, Lowy AM, et al. The American Society of Peritoneal Surface Malignancies evaluation of HIPEC with Mitomycin C versus Oxaliplatin in 539 patients with colon cancer undergoing a complete cytoreductive surgery. Journal of surgical oncology. 2014; 110: 779-785.

18. Votanopoulos K, Ihemelandu C, Shen P, et al. A comparison of hematologic toxicity profiles after heated intraperitoneal chemotherapy with oxaliplatin and mitomycin C. Journal of Surgical Research. 2013; 179: e133-e139.

19. Leung V, Huo YR, Liauw W, et al. Oxaliplatin versus Mitomycin C for HIPEC in colorectal cancer peritoneal carcinomatosis. European journal of surgical oncology: the journal of the European Society of Surgical Oncology and the British Association of Surgical Oncology. 2017; 43: 144-149.

20. Sparks DS, Morris B, Xu W, et al. Cytoreductive surgery and heated intraperitoneal chemotherapy for peritoneal carcinomatosis secondary to mucinous adenocarcinoma of the appendix. Int Surg. 2015; 100: 21-28. 